\title{
Stress-induced changes in the aged-rat adrenal cortex. Histological and histomorphometric study
}

\author{
S.M. Zaki'1, 2, F.A. Abdelgawad', E.A.A. El-Shaarawy'1, R.A.K. Radwan'1, B.E. Aboul-hoda' \\ ${ }^{1}$ Department of Anatomy and Embryology, Faculty of Medicine, Cairo University, Cairo, Egypt \\ ${ }^{2}$ Fakeeh College for Medical Sciences, Jeddah, Saudi Arabia
}

Received: 29 December 2017; Accepted: 1 March 2018]

Background: Stress exposure exerts direct effects on the morphology and functionality of the adrenal cortex. In addition, ageing effects growth, differentiation, apoptosis and cellularity of the cortex. The missing data is the combined effect of stress and ageing on the adrenal cortex. The aim of this study was to demonstrate the structural changes in the adrenal cortex following the exposure to stress in the adult and aged albino rats.

Materials and methods: Forty rats were divided into groups I and II (adult and senile). Each group was further subdivided into subgroups $a$ and $b$ (control and stressed). Light and electron microscopic studies were done. Area per cent of collagen fibres (Masson's trichrome-stained sections), number of proliferating cells (optical density immunoreactivity in the Ki67 stained sections) and thickness of the three adrenal zones were also measured.

Results: Lamellar separation of the capsule with subcapsular spindle cell hyperplasia and areas of ghost cells were observed in zona glomerulosa (ZG) and zona fasciculata (ZF) in group I-b. Separation and indentation of the capsule with its lamellar separation were observed in group II-a with the existence of multiple scattered degenerative foci in ZF and zona reticularis (ZR). Similar and aggressive was the architectural pattern of ZF in group II- $b$ with the presence of areas of homogenous degeneration. The nuclei of $Z G$ had marginated chromatin in group I-b and were pyknotic with deformed irregular outlines in group II-b. Multiple lysosomes and vacuolar degeneration mitochondria were also seen in group I-b. The nuclei of ZF were irregular with condensed marginated heterochromatin in group I-b, irregular with scattered chromatin in group II-a and indented with areas of chromatin destruction in group II-b. Mitochondria with disrupted cristae and cristolysis were also detected in group $1-b$. Numerous lipofuscin granules and dilated smooth endoplasmic reticulum were revealed in group II-b. The mean collagen fibre area per cent and the mean number of the proliferating cells in group II-b were significantly higher by $39 \%$ and $23 \%$. The thickness of ZG decreased significantly by $20 \%$ in group I-b. Contrary, the thickness of both ZF and ZR increased significantly by $10 \%$ in group $1-b$.

Conclusions: Histological alterations occurred in the adrenal cortex in response to stress, especially when coupled with the advance of age. This was accompanied by increase in the area per cent of collagen fibres and increase in the mean number of the proliferating cells in the adrenal cortex. (Folia Morphol 2018; 77, 4: 629-641)

Key words: stress, ageing, adrenal cortex 
Table 1. Experimental design

\begin{tabular}{llcc}
\hline Group & & Rat's age & Number of rats \\
\hline Group I (adult group) & Subgroup a (control group) & Reproductive senescence. Age of $10^{\text {th }}$ month on the basis of our & 10 \\
& Subgroup b (stressed group) & animal house colony & 10 \\
Group II (senile group) & Subgroup a (control group) & Post-senescence. Age of 21 & month on the basis of our animal \\
& Subgroup b (stressed group) & house colony & 10 \\
& & & 10 \\
\hline
\end{tabular}

\section{INTRODUCTION}

Stress is a state of disturbed homeostasis that allows the living organisms to adapt to environmental pressure. Stress responses are mainly mediated by the activation of the hypothalamic-pituitary-adrenal axis [22]. The adrenal gland is the primary peripheral endocrine gland in the stress response [16]. It is composed of two functionally discrete organs, the cortex which synthesizes steroid hormones that mediate body homeostasis and chronic stress responses and the medulla which produces catecholamines that facilitate acute stress [27]. Stress exposure can exert direct effects on the morphology as well as the functionality of the adrenal gland [1].

Ageing of the human adrenal cortex presents several problems such as changes in growth, differentiation; apoptosis and cellular senescence affect the properties of this tissue over the lifespan [13]. The different layers of the adrenal cortex undergo changes with ageing, including a reduction in the thickness of the zona reticularis (ZR) and a relative increase of the thickness of the outer cortical zones. With the advance of age, the cortex begins to show microscopic nodular changes [3]. Spindle or fusiform cell proliferation beneath the adrenal capsule is a common finding in mice with the advance of age [9].

The missing data is the combined effect of stress and ageing on the adrenal cortex. So, the aim of the present work was to demonstrate the histological, morphometric as well as the immunohistochemical changes occurring in the adrenal cortex following exposure to stress in the adult and aged albino rats.

\section{MATERIALS AND METHODS}

\section{Animals}

Forty Wister male albino rats were divided into two groups. Each group was further subdivided into two subgroups. The rats were sacrificed after 14 days. The study was approved by the Ethics Committee, Faculty of Medicine, Cairo University (431/2017). All animal procedures followed the ethical guidelines of experimental animals. We followed the guidelines of the ethical standards of the National Institutes of Health guide for the care and use of Laboratory Animals (NIH Publications No. 8023, revised 1978).

\section{Experimental design}

Experimental design is shown in Table 1. Rats in subgroup a were housed separately in plastic restrainers $(6 \mathrm{~cm}$ wide $\times 12 \mathrm{~cm}$ long) for $2 \mathrm{~h}$ daily for 14 days [24].

\section{Light microscopic examination}

The specimens were obtained from the adrenal gland of each rat of all groups. They were kept in $10 \%$ formol saline, processed for paraffin sections of 5-7 $\mu \mathrm{m}$ thickness and mounted on glass slides for histopathological examination. The sections were stained with haematoxylin and eosin (H\&E) stain to study the histological changes and stained with Masson's trichrome stain to demonstrate the collagen fibres.

\section{Immunohistochemical staining}

The Ki67 antigen (a cell division marker) was used to detect cell proliferation in the adrenal cortex. Immunohistochemistry for Ki67 antigen detects cells that are in late $\mathrm{G} 1, \mathrm{~S}, \mathrm{G} 2$, and $\mathrm{M}$ but not $\mathrm{G} 0$ phases of the cell cycle [5].

The technique was done according to the manufacturer's instructions. The glands were mounted on polysine slides. Endogenous peroxidase activity was blocked by immersing sections in 3\% hydrogen peroxide in methanol for $30 \mathrm{~min}$ in the dark at room temperature. The sections were then rehydrated and washed in phosphate buffered saline (PBS; 2-5 min). To unmask antigenic sites, sections were microwaved at full power for 4-5 $\mathrm{min}$ in $0.01 \mathrm{M}$ sodium citrate buffer, $\mathrm{pH}$ 6.0, and then cooled and washed in PBS (5 $\mathrm{min}$ ). The sections were treated with blocking serum $(20 \%$ normal rabbit serum in $1 \times$ Tris-buffered saline [TBS], pH 7.6) for $30 \mathrm{~min}$. Then, the sections were treated with the primary anti Ki67 anti- 
body (Novocastra Lab Ltd.; NCL-Ki67-MM1) for $2 \mathrm{~h}$. Biotinylated secondary antibody (rabbit anti-mouse IgG biotin, Dako E0354) was applied for $30 \mathrm{~min}$. Then, the sections were treated with avidin-biotin-horseradish peroxidase solution ( $A B$ Complex/HRP kit; Dako K0355) for 30 min at room temperature. Finally, the sections were incubated for 3 min with 3,3'-diaminobenzidine solution (DAB isopac, Sigma D9015), comprising $25 \mu \mathrm{L}$ DAB $(2 \mathrm{mg} / \mathrm{mL})+5.9 \mathrm{~mL} 0.2$ MTBS $+1 \mu \mathrm{L} 30 \% \mathrm{H}_{2} \mathrm{O}_{2}$. After lightly counterstaining with haematoxylin (Vector, H3401), slides were rinsed, dehydrated through graded ethanol, cleared in xylene and mounted under coverslips with DPX mounting medium. The cells were considered positive for the Ki67 antigen when the nuclei were stained with a brownish colour [6].

\section{Electron microscopic examination}

Specimens from the adrenal cortex were fixed in $4 \%$ glutaraldehyde then washed in phosphate buffer and post-fixed in $1 \%$ osmium tetraoxide. Fixation was followed by dehydration and embedding in epoxy resins. Semithin sections $(1 \mu \mathrm{m})$ were stained with toluidine blue. Ultrathin sections (50-60 nm) were stained with uranyl acetate and lead citrate. These sections were examined and photographed using a Joel, $100 \mathrm{CX}$ II transmission electron microscope.

\section{Histomorphometric studies}

Quantitative data were obtained using "Leica Qwin 500 C" image analyser computer system Ltd. (Cambridge, England). The following parameters were measured in different fields with fixed field area $4 \times 10^{4} \mu \mathrm{m}^{2}$ : area per cent of collagen fibres (Masson's trichrome-stained sections), the number of proliferating cells (optical density immunoreactivity in the Ki67 stained sections) and the thickness of the three adrenal zones.

\section{Statistical analysis}

Statistical analysis was performed using statistical package for the social sciences (SPSS) version 21.0 (IBM Corporation, Somers, NY, USA) statistical software. The data were expressed as means \pm standard deviation (SD). Statistical evaluation was done using one-way analysis of variance (ANOVA) followed by Bonferroni pairwise comparisons. Significance was considered when $p$-value was $\leq 0.05$. The percentage of increase or decrease (difference) of all study parameters were calculated per the following formula:

$\begin{aligned} & \text { Mean difference value between } \\ & \text { difference }\end{aligned}$
two groups

\section{RESULTS}

Histological section observations

Zona glomerulosa (ZG) cells were arranged in clusters, zona fasciculata (ZF) cells were arranged in straight columns and ZR cells were arranged as anastomosing cords in group I-a (Fig. 1a). Lamellar separations of the capsule with areas of ghost cells were observed in ZG and ZF in group I-b (Fig. 1b, c). Separation and indentation of the capsule with its lamellar separation were observed in group II-a. In addition, the arrangement of ZG cells was disturbed with the existence of multiple scattered degenerative foci in ZF and ZR (Fig. 1d-f). Capsular separation with subcapsular spindle cell hyperplasia and disruption of the regular architectural pattern of ZF were seen in group II-b with the presence of areas of homogenous degeneration (Fig. 1g, h).

Spindle cell hyperplasia and vacuolated cell-areas were detected in ZR in the group I-b (Fig. 2a, b). Scattered brown granules, wide sinusoids, and areas of degeneration in ZR were observed in group II-a (Fig. 2c, d). The disturbed arrangement of the ZR cells with engorged central adrenal veins and congested sinusoids were seen in group II-b (Fig. 2e, f).

\section{Semithin sections findings}

Thickening with lamellar separation of the capsule with irregular shape and arrangement of its fibroblasts were observed in group II-b. ZG cells exhibited lipid droplet-loaded cytoplasm in the groups I-a, I-b, II-a. Their nuclei exhibited different shapes and sizes in group I-b, darkly stained in group II-a, pyknotic in group II-b (Fig. 3). The nuclei of ZF were rounded vesicular in groups I-a, II-a, while they were irregular in shape and darkly hyperchromatic in group II-b (Fig. 4).

\section{Masson's trichrome-stained sections and immunohistochemical study}

The capsule was regular with parallel arranged collagen fibres in group I-a, while it was disrupted with thickened fibrous septa in group I-b. Lamellar separation of the capsule with irregularly dispersed collagen fibres was seen in group II-a. The capsule 


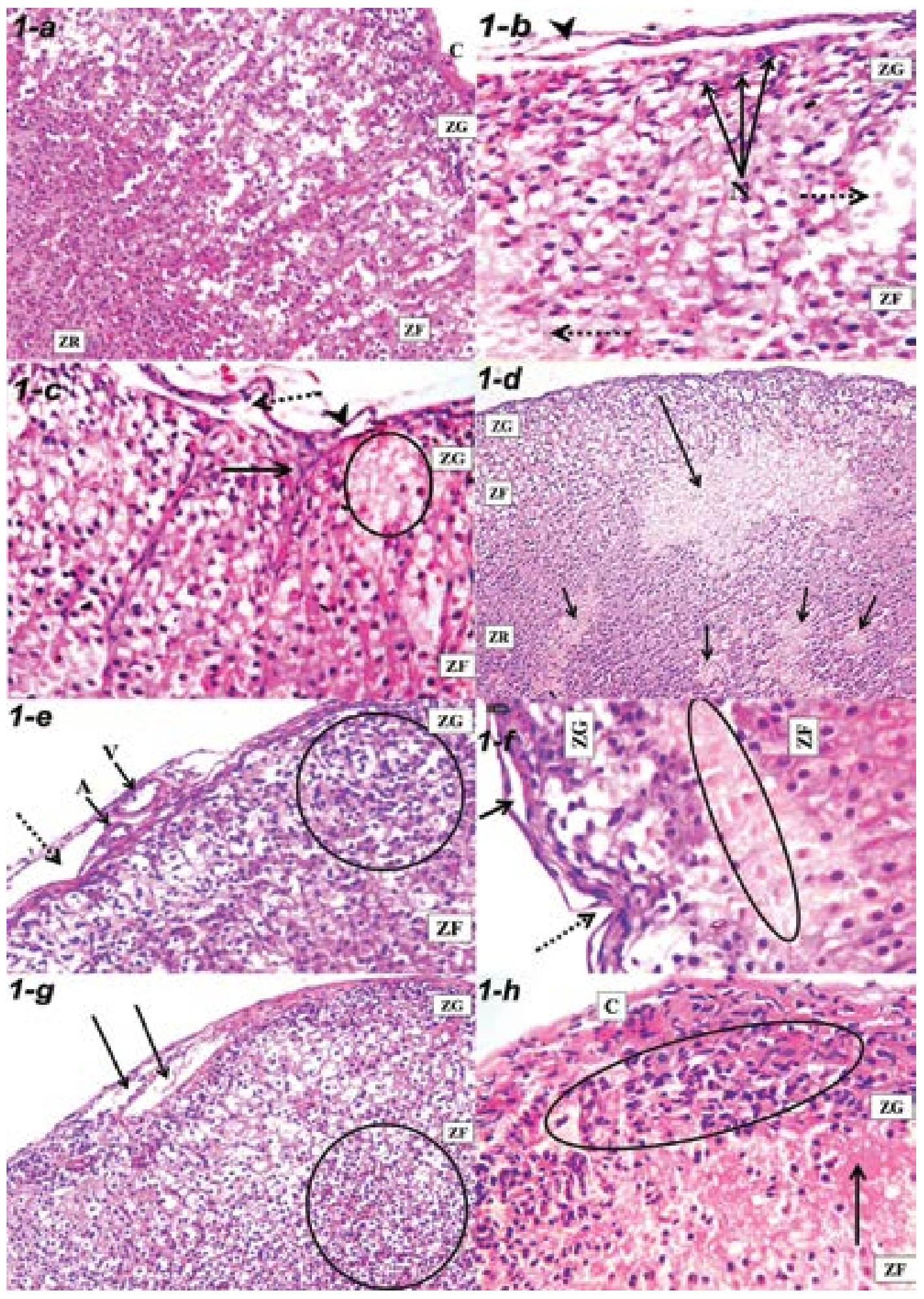

Figure 1. Sections of the ZG and ZF (H\&E); a. Capsule (C), ZG cells arranged in clusters, ZF cells arranged in straight columns and ZR cells arranged as anastomosing cords in a rat group $\mathrm{I}-\mathrm{a}(\times 200)$. b. Capsular separation (arrowhead), multiple pleomorphic nuclei $(\mathrm{N})$ and areas of ghost cells (dotted arrows) in ZF in a rat group I-b $(\times 400)$. c. Lamellar separation of the capsule (dotted arrow), thin overlying capsule (arrowhead) and subcapsular spindle cell hyperplasia (arrow). Note an area of ghost cells (circle) in ZG in a rat group I-b ( $\times 400)$. d. Multiple scattered degenerative foci in the ZF (long arrow) and ZR (short arrows) in a rat group II-a $(\times 100)$. e. Capsular separation (dotted arrow) with the disturbed arrangement of ZG cells (circle). Note a capsular artery (A) and vein (V) in a rat group II-a $(\times 200)$. f. Capsule with lamellar separation (arrow) and indentation (dotted arrow). Note an area of degenerated ghost cells (circle) in a rat group II-a $(\times 400)$. g. Capsular separation (arrows) with disruption of the regular architectural pattern (circle) of ZF in a rat group II-b $(\times 150)$. h. Capsule (C) with subcapsular spindle cell hyperplasia (circle). Note: an area of homogenous degeneration (arrow) in a rat group II-b $(\times 400) ; \mathrm{ZG}$ — zona glomerulosa; ZF — zona fasciculata; ZR — zona reticularis. 


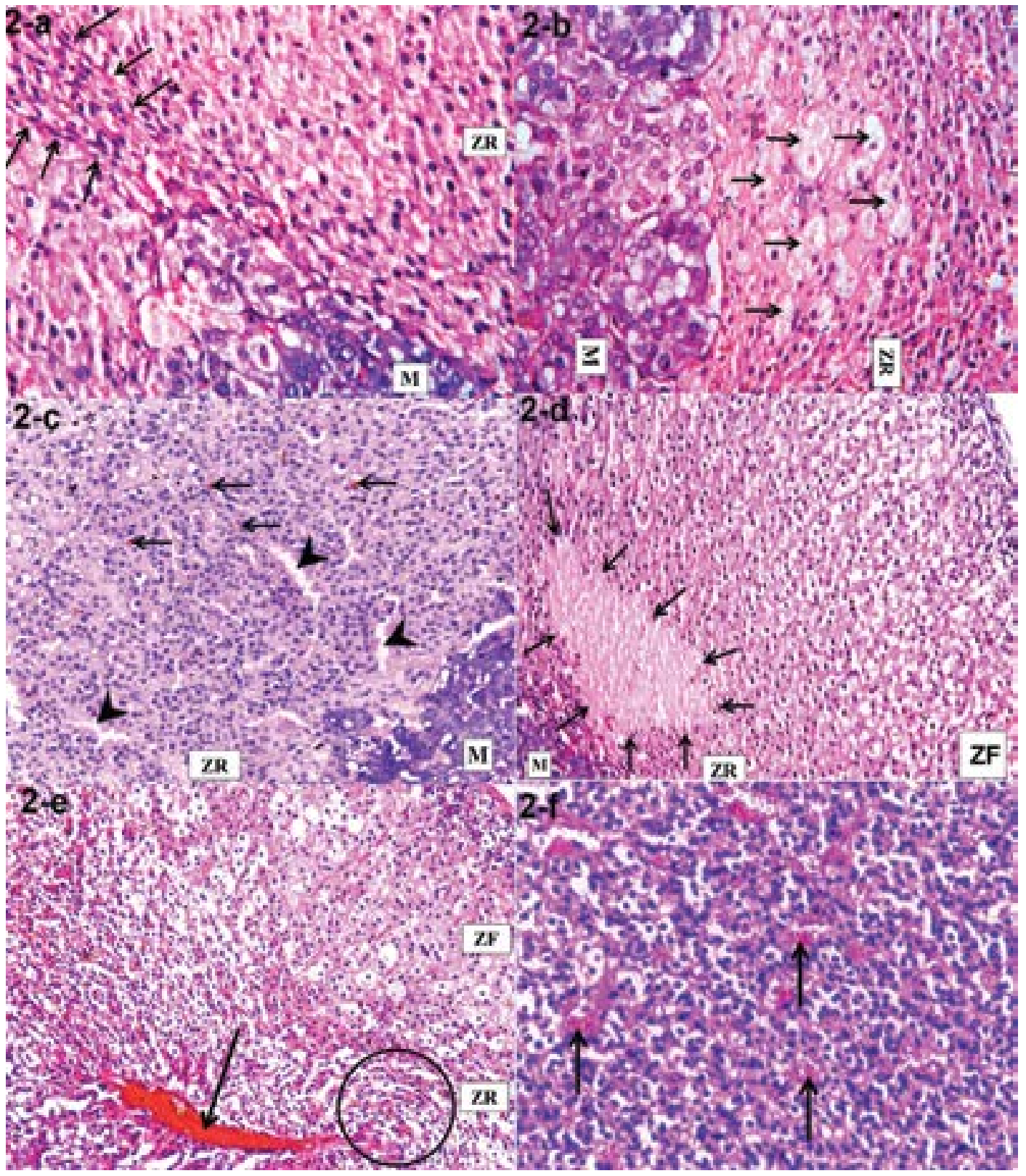

Figure 2. Sections of the zona reticularis (ZR) and adjoining medulla (M) (H\&E); a. Spindle cell hyperplasia (arrows) in a rat group I-b $(\times 400)$. b. An area of vacuolated cells (arrows) in a rat group I-b $(\times 400)$. c. Scattered brown granules (arrows) and wide sinusoids (arrowheads) in a rat group II-a $(\times 400)$. d. An area of degeneration (arrows) in a rat group II-a $(\times 150)$. e. A disturbed arrangement (circle) of the ZR cells and an engorged central adrenal vein (arrow) in a rat group II-b $(\times 150)$. f. A congested sinusoids (arrows) in a rat group II-b $(\times 400)$.

was thickened with lamellar separation and thickened disrupted collagen bundles in group II-b. Dilated congested blood capillaries with extravasation of blood and exudation were also seen in group II-b (Figs. 5, 6).

The immunoreaction of Ki67 was nuclear. It was absent in groups $\mathrm{I}-\mathrm{a}, \mathrm{II}-\mathrm{a}$, while it was positive in groups I-b, II-b (Fig. 7).

\section{Ultrastructural remarks}

The nuclei of ZG were euchromatic with prominent nucleoli in groups I-a and II-a, had marginated chro- matin in group I-b and were pyknotic with deformed irregular outlines and disrupted nuclear envelops in group II-b. Multiple lysosomes and vacuolar degeneration mitochondria were also seen in group I-b (Fig. 8).

The nuclei of ZF were regular and rounded in group I-a, irregular with condensed marginated heterochromatin in group I-b, irregular with scattered chromatin in group II-a and indented with areas of chromatin destruction in group II-b. Mitochondria with disrupted cristae and discontinuity of their envelopes and mitochondria with cristolysis and loss 


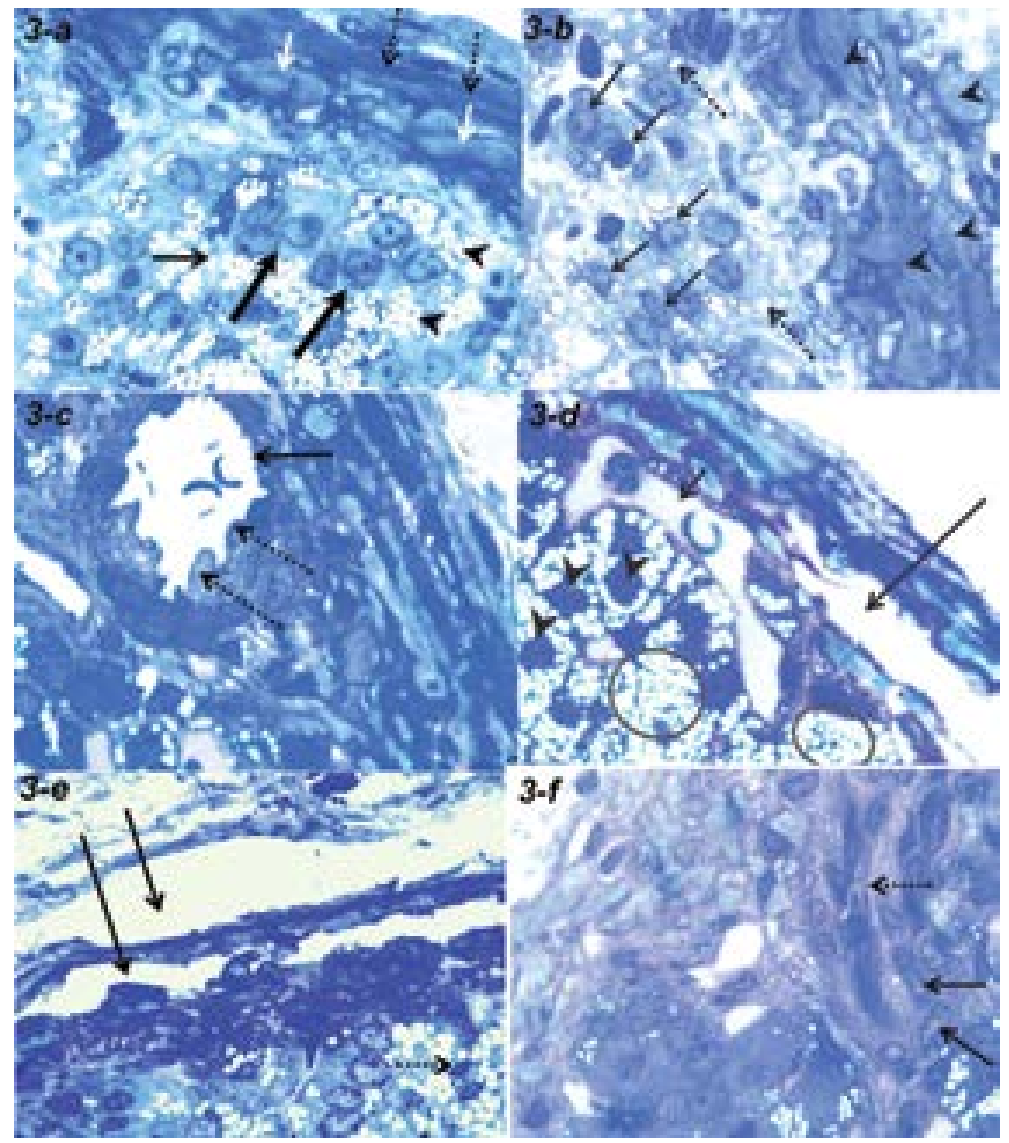

Figure 3. Semithin sections of the zona glomerulosa (ZG) and zona fasciculata (ZF) (Toluidine blue $\times 1000)$; a. Capsular connective tissue fibres (dotted arrows), fibroblasts (white arrows) and ZG cells (thick black arrows) with the cytoplasm loaded with lipid droplets (arrowheads) in a rat group l-a. Note a blood capillary (thin black arrow). b. Large swollen disrupted arrangement fibroblasts (arrowheads). The nuclei of the ZG cells exhibited different shapes and sizes (arrows), while the cytoplasm was loaded with lipid droplets (dotted arrows) in a rat group I-b. c. A distended capillary (arrow) with folded endothelium (dotted arrows) in a rat group II-a. d. Capsular separation (long arrow) and a markedly dilated blood vessel (short arrow). The ZG shows abundant lipid droplets (circles) and darkly stained nuclei (arrowheads) in a rat group II-a. e. A lamellar separation of the capsule (arrows) and ZG with pyknotic nuclei (dotted arrow) in a rat group II-b. f. A marked thickening of the capsule with irregular shape and arrangement of its fibroblasts (arrows) and collagen fibres (dotted arrow) in a rat group II-b.

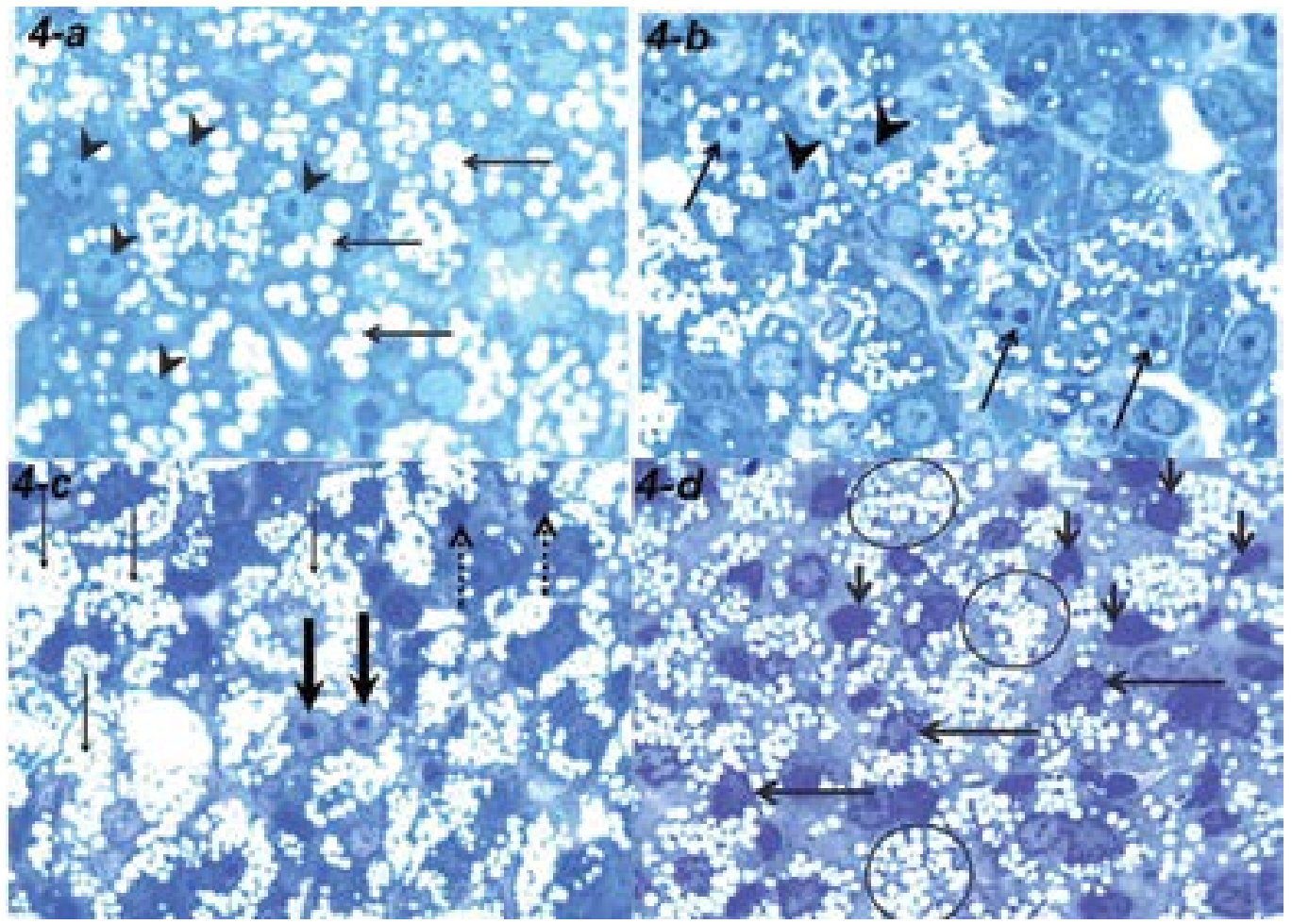

Figure 4. Semithin sections of the zona fasciculata (ZF) and zona reticularis (ZR) (Toluidine blue $\times 1000)$; $a$. ZF with rounded vesicular nuclei (arrowheads) and lipid droplets loaded cytoplasm (arrows) in a rat group I-a. b. ZR with rounded nuclei (arrowheads) and prominent nucleoli. Note nuclei with double nucleoli (arrows) in a rat group I-b. c. ZF with vesicular regular shaped nuclei (thick arrows) and lipid droplets loaded cytoplasm (thin arrows) in a rat group II-a. Note pyknotic nuclei (dotted arrows). d. ZF with irregular shape nuclei (long arrows), darkly stained hyperchromatic nuclei (short arrows) in a rat group II-b. Note lipid droplets (circles). 


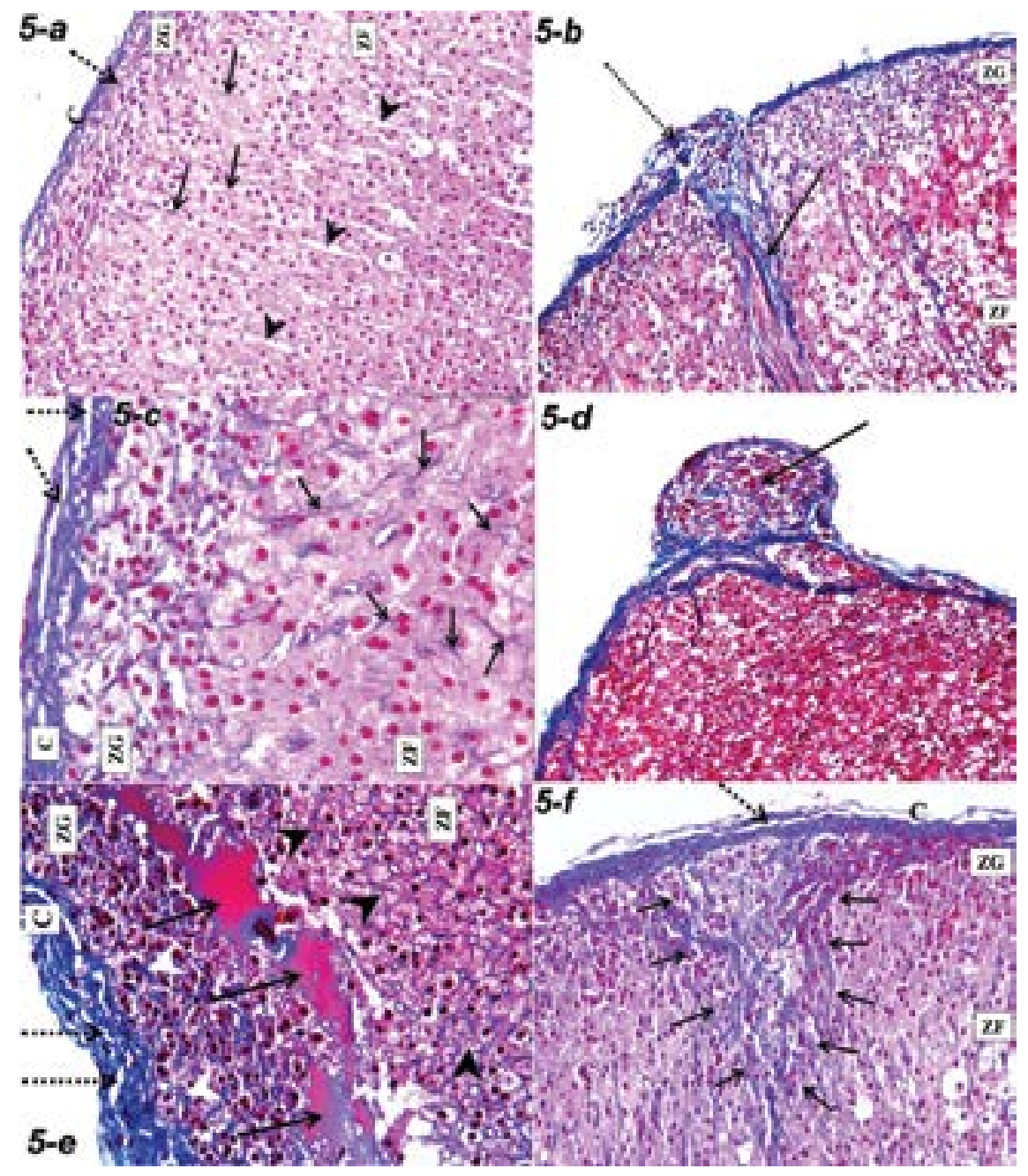

Figure 5. Collagen fibres in zona glomerulosa (ZG) and zona fasciculata (ZF) (Masson's trichrome); a. A regular capsule (C), trabeculae of collagen fibres (dotted arrow) and parallel arranged collagen fibres (arrows) perpendicular to the surface in a rat group l-a. Note blood sinusoids (arrowheads) ( $\times 200)$. b. A thickened fibrous septum (arrow) and a disrupted area in the capsule (dotted arrow) in a rat group I-b $(\times 200)$. c. Lamellar separation (dotted arrows) of the capsule $(C)$ and irregularly dispersed collagen fibres (arrows) in a rat group II-a $(\times 400)$. d. Accessory adrenocortical tissue (arrow) in a rat group II-a $(\times 100)$. e. Thickened-capsule $(C)$ with lamellar separation of its collagen bundles (dotted arrows), thickened collagen bundles (white arrowheads), irregular collagen fibres (black arrowheads) and areas of exudate (arrows) in a rat group II-b $(\times 400)$. f. A thickened fibrous septum (arrows) and a thickened partially separated capsule (dotted arrow) in a rat group II-b $(\times 200)$.

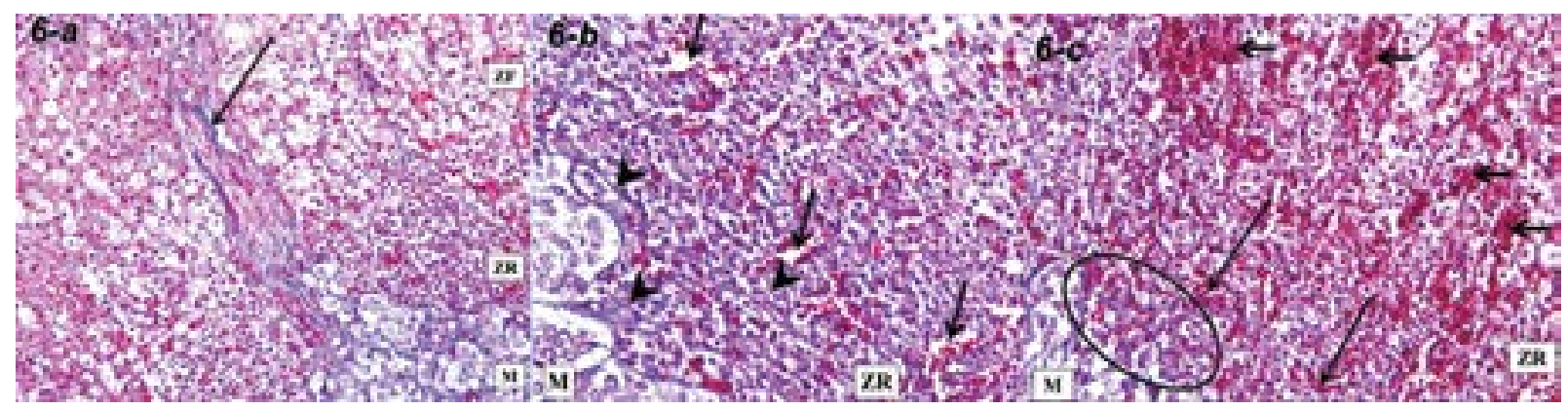

Figure 6. Collagen fibres in zona fasciculata $(\mathrm{ZF})$, zona reticularis $(\mathrm{ZR})$, and adjoining medulla $(\mathrm{M})$ (Masson's trichrome $\times 200)$; a. Thickened collagen bundles (arrow) in a rat group l-b. b. A network of collagen bundles (arrowheads) and numerous blood capillaries (arrows) in a rat group II-a. c. A thickened disrupted collagen bundles (circle) dilated congested blood capillaries (long arrows) with extravasation of blood (short arrows) in a rat group II-b. 


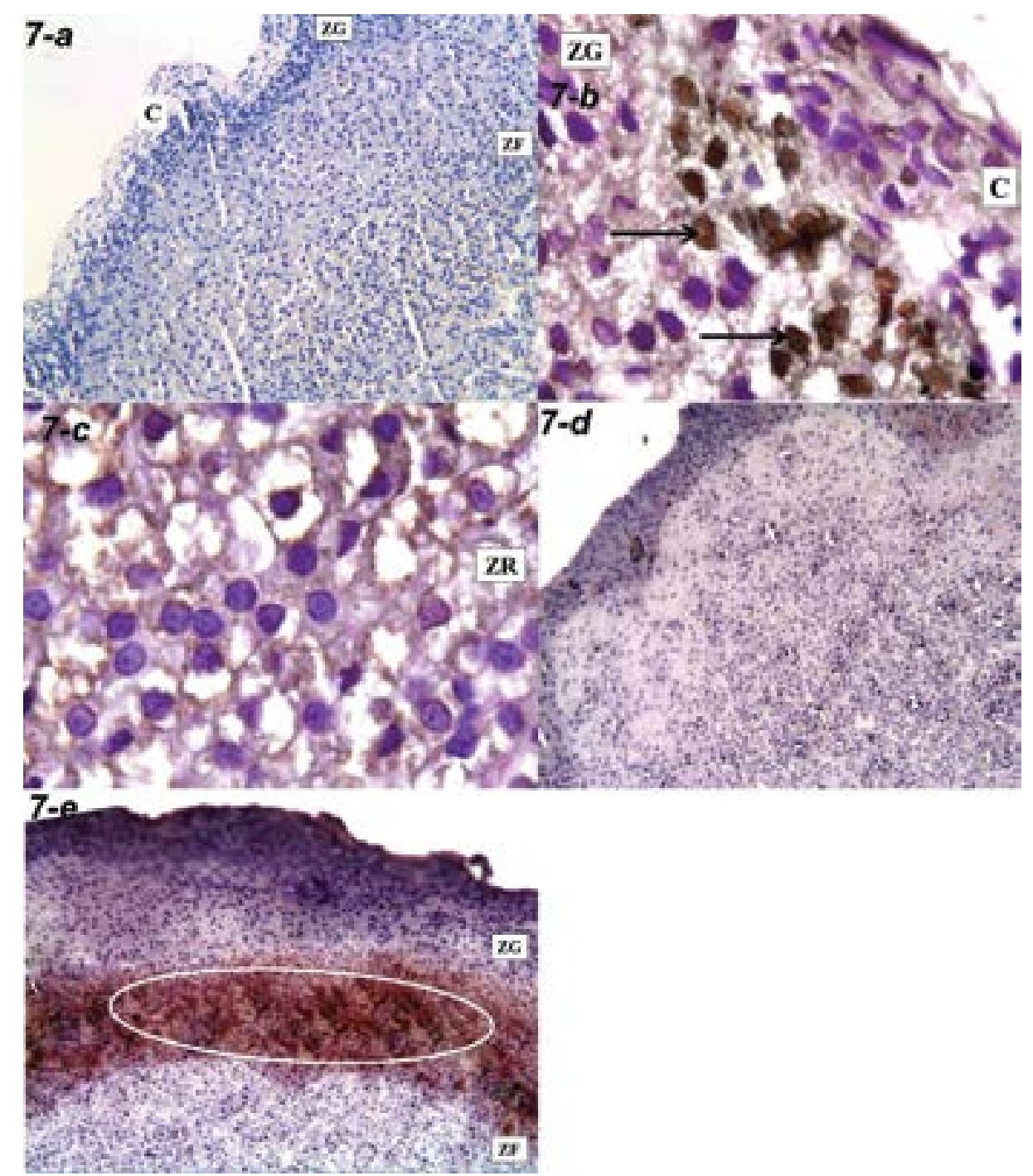

Figure 7. Immunoreactivity in the different groups (Ki67); a. Absence of immunoreactivity in a rat group $\mathrm{I}-\mathrm{a}(\times 200)$. b. Immunoreactive nuclei (arrows) in a rat group I-b $(\times 1000)$. c. Absence of immunoreactive nuclei in the ZR in a rat group I-b $(\times 1000)$. d. Absence of immunoreactivity in a rat group II-a $(\times 200)$. e. Area of immunoreactivity (white circle) at the junctional zone between ZG and ZF in a rat group II-b $(\times 200)$; ZG — zona glomerulosa; ZF — zona fasciculata; ZR — zona reticularis.

of their envelopes were also detected in group $\mathrm{I}-\mathrm{b}$ (Fig. 9).

The absence of nuclear affection was perceived in the ZR cells of the different groups. Lipofuscin granules and dilated smooth endoplasmic reticulum were also observed in group II-b (Fig. 10).

\section{Morphometric measurements (Tables 2-4)}

The mean collagen fibre area per cent of group II-b was $39 \%$, significantly higher than that of group II-a. Using the optical density of the Ki67, the mean number of group II-b proliferating cells was significantly higher by $23 \%$ than that of group I-b.

The thickness of ZG decreased significantly by $20 \%$ in group I-b as compared to that of group I-a.
Contrary, the thickness of both ZF and ZR increased significantly by $10 \%$ in group $\mathrm{I}-\mathrm{b}$ as compared to their respective of group I-a.

\section{DISCUSSION}

Stress is a state of endangered homeostasis. The effect of stress on the adrenal cortex was evaluated as the adrenal gland is the chief stress responsive organ involved in the overall homeostasis. Various types of experimental stressors have been styled such as restraint, cold/heat, water immersion, water deprivation, noise, crowding and electric shock [10]. Among all these methods, restraint is the method that attains the least pain, suffering or distress to the animal. 


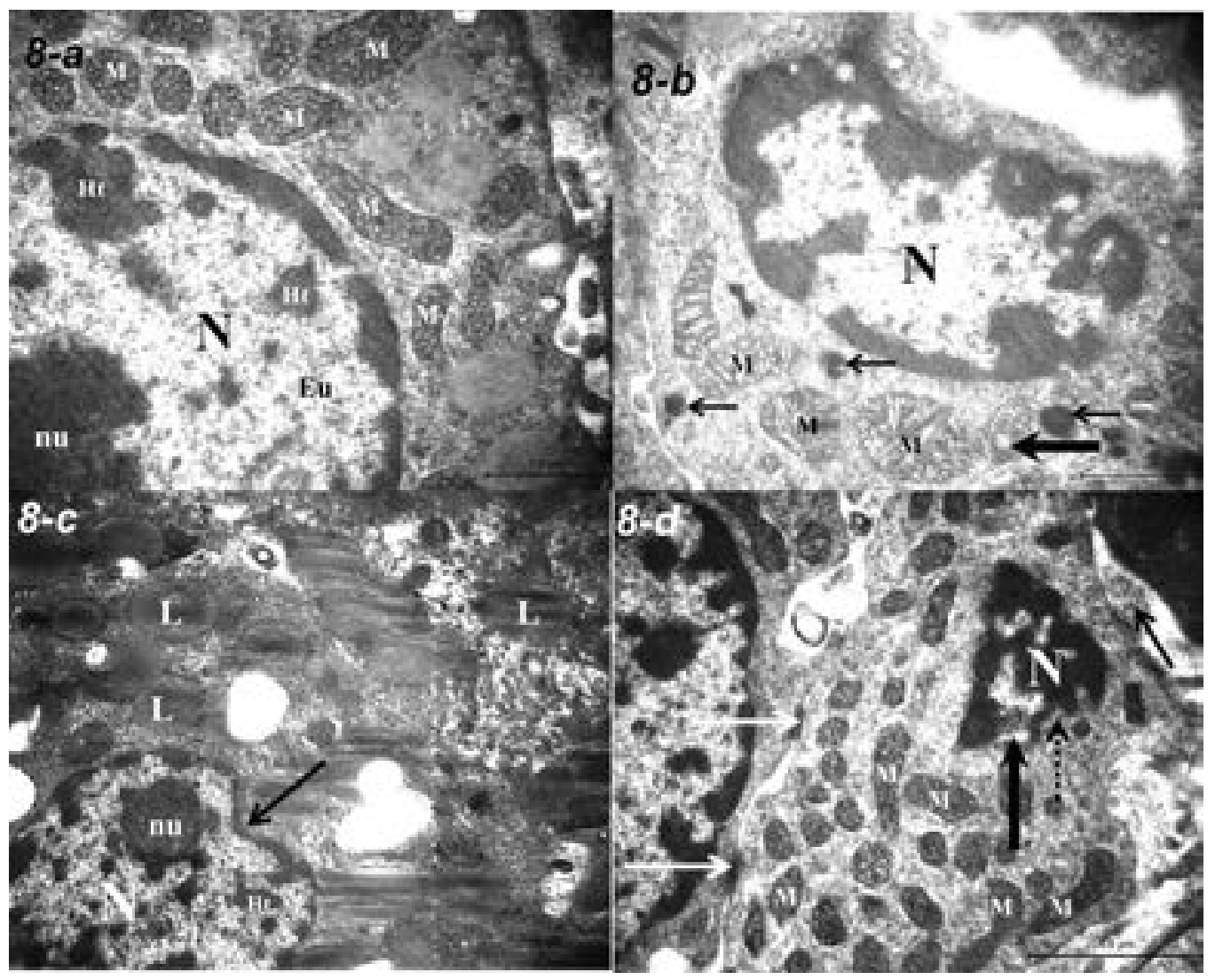

Figure 8. Electron photomicrograph of the zona glomerulosa; a. A nucleus (N) containing euchromatin (Eu), clumps of peripheral heterochromatin $(\mathrm{Ht})$, prominent nucleolus (nu) and multiple elongated mitochondria (M) with tubulolamellar cristae in a rat group l-a (TEM $\times 20000)$. b. A nucleus (N) with clogged marginated deeply stained chromatin distorting the nuclear contour. The cytoplasm contains multiple lysosomes (arrows), mitochondria (M) with tubulolamellar cristae and mitochondria with vacuolar degeneration (thick arrow) in a rat group I-b $(T E M \times 20,000)$. c. A nucleus $(\mathrm{N})$ with distinct nucleolus $(\mathrm{nu})$, heterochromatin $(\mathrm{Ht})$ and undulation of its nuclear envelope (black arrow) in a rat group II-a. Note lipid droplets (L) (TEM $\times 10,000)$. d. A pyknotic nucleus (N) with a deformed irregular outline (dotted arrow), dense clumps of clogged chromatin associated with disrupted nuclear envelope (thick arrow) in a rat group II-b. Note mitochondria (M), desmosomes (white arrows) and bundles of collagen fibres (black arrow) (TEM $\times 15,000)$.

The present study was confined to male rats since the adrenal gland of female rats undergo several changes such as the appearance and regression of the X-zone [9] and the response to stress is a sex-specific due to a gender difference in the hormonal receptors [2].

Areas of subcapsular spindle cell hyperplasia were detected in the adult and senile stressed groups (groups I-b, II-b). These cellular clusters were located initially in the ZG and then extend centripetally between the underlying ZF cell cords. Several explanations had been suggested: a mast cells role [15], sex hormones association [20] or misspecifications of subcapsular mesenchymal stem cells that do not differentiate to enter the steroidogenic lineage [28].

Ghost cell areas, ghost nuclei, multiple pleomorphic and pyknotic nuclei existed in ZG of the adult stressed group. The nuclei of ZG cells are extremely sensitive to stress [16]. Areas of vacuolated cells at the junctional zone between ZR and medulla existed in this group. Stress induces pronounced changes at the interphase between ZR and medulla where connective tissue cords loosened, cell count decreased and cell-to-cell spaces extended [18]. These vacuolated areas might denote an early stage of cystic degeneration [21].

A significant decrease in the thickness of the cortical zones was observed in the adult stressed group (group I-b) which may be related to shifting in hormonal stress responsiveness [7].

Increase thickness, indentation with lamellar separation were constant findings of the capsule of the senile control group (group II-a). In addition, replacement of the capsular cellular elements with collagenous tissue was also detected; such replacement was suggested as a mechanism responsible for the age-dependent capsular changes [14].

Disturbance of the arrangement the ZG was noticeable in both senile groups (groups II-a, II-b). The 


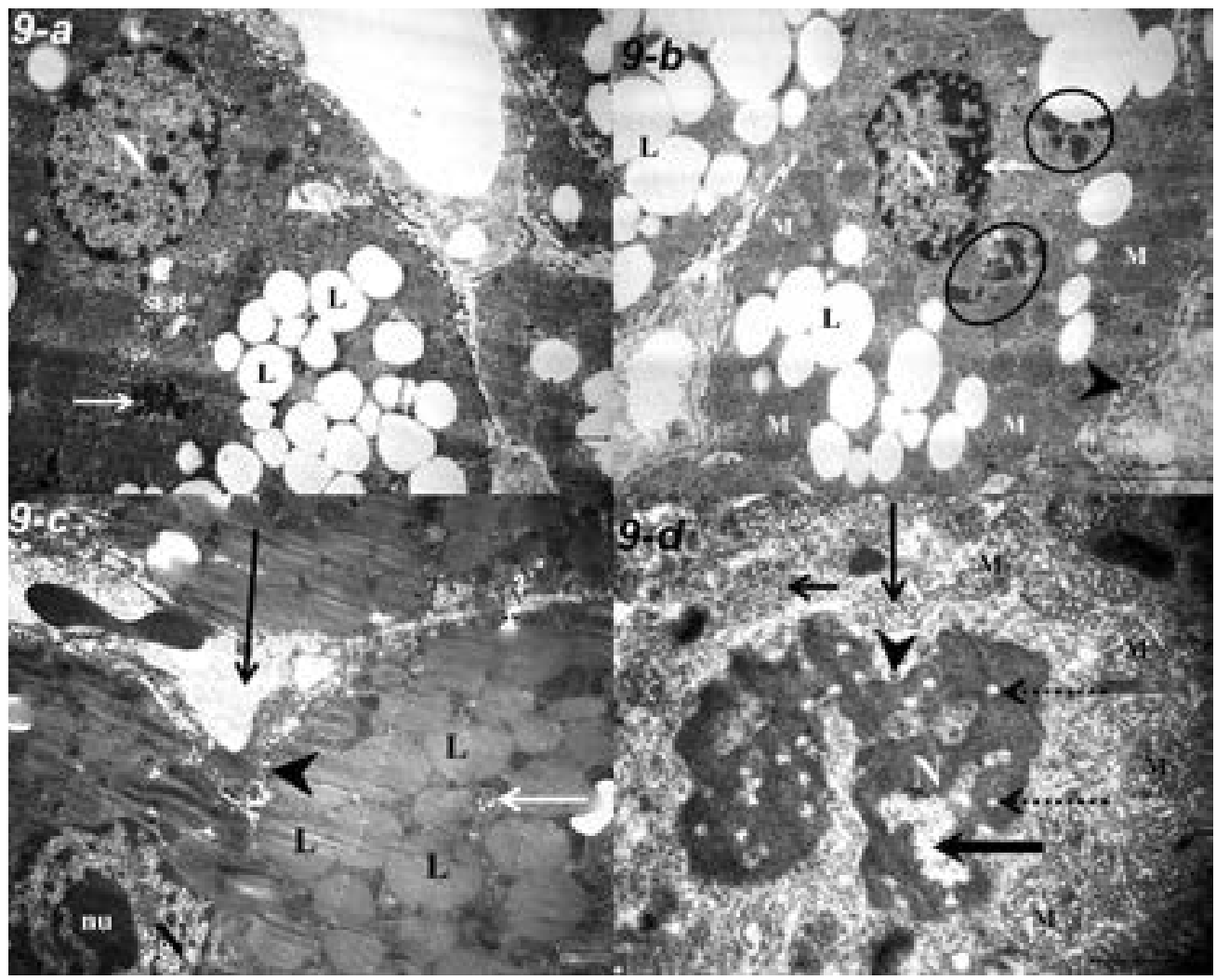

Figure 9. Electron photomicrograph of the zona fasciculata; a. A regularly rounded nucleus (N), several lipid droplets (L), smooth endoplasmic reticulum and lipofuscin granules (white arrow) in a rat group I-a (TEM $\times 8000)$. b. A nucleus $(\mathrm{N})$ with irregular outline, condensed marginated dense clumps of heterochromatin (white arrow), lipofuscin granules (circles), many mitochondria (M), multiple variable sized lipid droplets (L) and microvilli (arrowhead) on the cell surface membrane in a rat group I-b $($ TEM $\times 10000)$. c. A nucleus $(\mathrm{N})$ with irregular outline containing scattered chromatin and prominent nucleolus (nu).The cytoplasm contains secondary lysosome (white arrow), electron-dense lipid droplets (L). Note a wide blood sinusoid (black arrow) and microvilli (arrowhead) in a rat group II-a (TEM $\times 8000$ ). d. A highly indented bilobed nucleus (N) with the undulation of its nuclear envelope (arrowhead), electron-lucent spots (dotted arrows) and focal areas of chromatin destruction (thick arrow) in a rat group II-b. Note mitochondria (M) with tubulolamellar cristae, mitochondria with disrupted cristae, discontinuity mitochondrial envelope (long arrow), mitochondria with cristolysis and loss of mitochondrial envelope (short arrow) (TEM $\times 20,000)$.

imperfect repair of damage with the advance of age combined with compensatory overgrowth can lead to more and more abnormal tissue architecture [12]. In addition, increased vacuolation of the ZG cells reflected a decrease in the secretory activity of ZG cells [25]. The three cortical zones normally have some degree of vacuolation; these vacuoles contain neutral lipid and cholesterol. The increased cortical vacuolation can be due to age-dependent drop in cholesterol biosynthesis or metabolism leading to accumulation of cholesterol and steroids [25].

Areas of degeneration with a cytoplasmic heterogeneity of the ZF cells was seen in group II-a which reflects different cell sensitivity to destructive factors and an increase in the subpopulation of newly formed adrenocortical cells [17]. Another notable finding in this group was the predominance of lipofuscin-like inclusions seen in the cytoplasm of the ZR cells. Lipofuscin is a biomarker of ageing and its progressive accumulation is one of the most prominent changes with ageing [14]. Deposition of lipofuscin pigment is most probably due to decreased metabolic activities in the cells [14]. It accumulates gradually in lysosomes of post-mitotic tissue and it is not removed by degradation or exocytosis [4].

Accessory adrenocortical tissue was also encountered in group II-a. This tissue is composed of normal cortical tissue, in which ZG and ZF are notable while the medullary tissue is not normally associated [11]. The accessory cortical tissues undertake the same ageing changes as the main body of the adrenal [11].

The senile stressed group (group II-b) exhibited marked disruption of the normal architecture of three adrenocortical zones which may be related to the effect of elevated corticosterone level [8]. This disturbance was associated with different vascular changes in the form of patches of exudate in $\mathrm{ZF}$, congestion of the capillaries in $\mathrm{ZR}$ and marked 


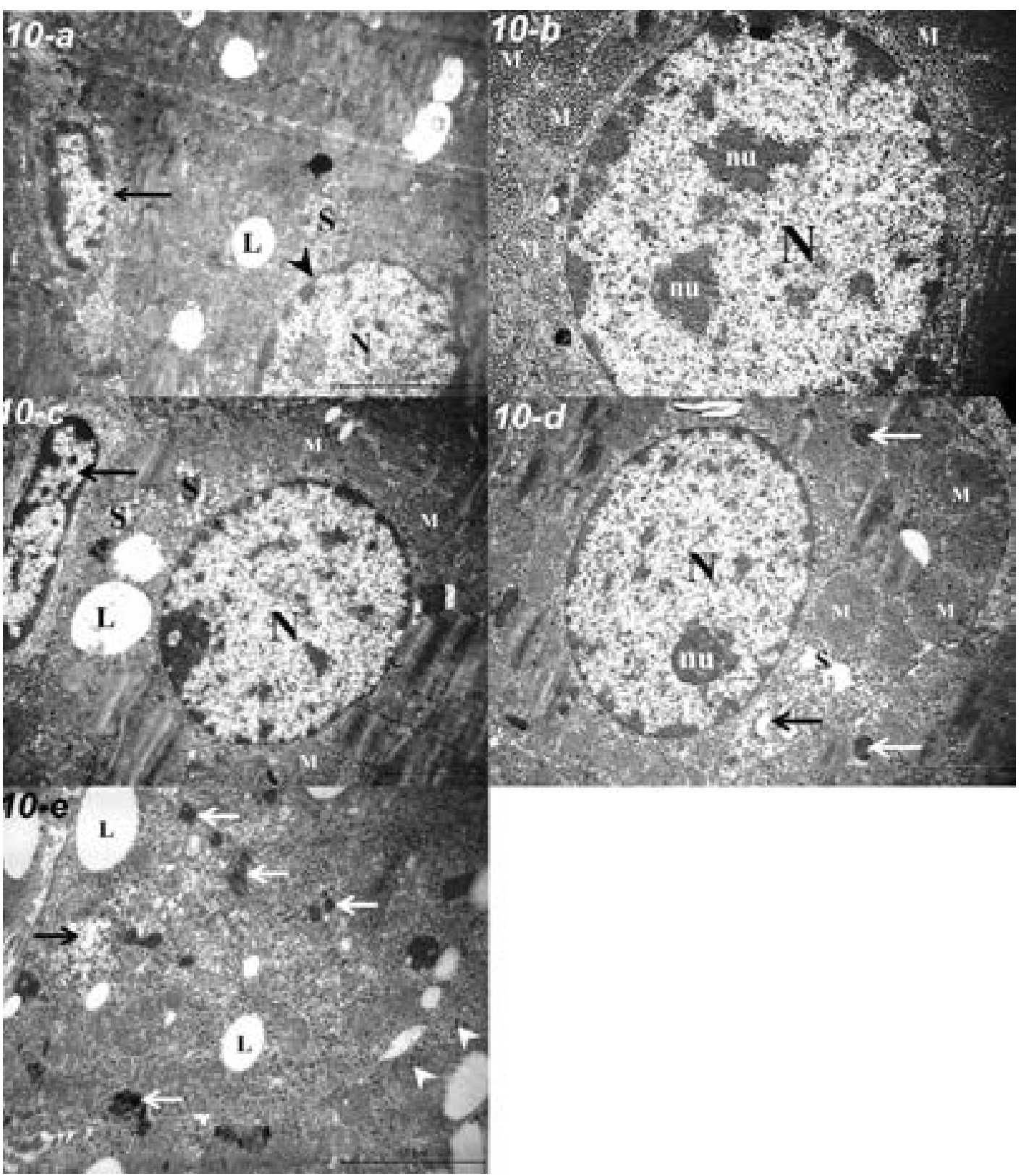

Figure 10. Electron photomicrograph of the zona reticularis; a. A euchromatic nucleus (N) with a nuclear envelope (arrowhead), a profile of smooth endoplasmic reticulum (SER) (S), lipid droplets (L) and a spindle-shaped fibroblast (arrow) in a rat group l-a $(T E M \times 10,000)$. b. A rounded euchromatic nucleus (N) possessing double nucleoli (nu) and mitochondria (M) with numerous tubular cristae in a rat group I-b $(T E M \times 15,000)$. c. a euchromatic nucleus (N), SER $(S)$, multiple mitochondria $(M)$ and few lipid droplets $(L)$ in a rat group II-a. Note an elongated endothelial nucleus (black arrow) (TEM $\times 10,000$ ). d. A euchromatic nucleus (N) with a distinct nucleolus (nu), mitochondria (M), dilated SER (S), lysosomes (white arrows), and Golgi body (black arrow) in a rat group II-b (TEM $\times 10,000)$. e. Few lipid droplets (L), numerous lipofuscin granules (white arrows), dilated SER (black arrow) and free ribosomes (arrowheads) in a rat group II-b (TEM $\times 20,000)$.

Table 2. Area per cent of collagen fibres

\begin{tabular}{lcc}
\hline Groups & Mean \pm SD & P \\
\hline Group I-a & $31.6 \pm 4.7$ & 0.09 \\
Group I-b & $38.7 \pm 0.9$ & \\
Group II-a & $41.4 \pm 3.7$ & $0.02^{*}$ \\
Group II-b & $57.8 \pm 2.2$ & \\
\hline
\end{tabular}

${ }^{*}$ Significant $(\mathrm{p} \leq 0.05)$; SD - standard deviation
Table 3. Optical density of Ki67 immunoreactivity in the stressed groups

\begin{tabular}{lll}
\hline Groups & Mean \pm SD & P \\
\hline Group I-b & $67.46 \pm 5.17$ & $0.02^{*}$ \\
Group II-b & $83.05 \pm 1.63$ & \\
\hline
\end{tabular}

${ }^{*}$ Significant $(p<0.05)$; SD — standard deviation 
Table 4. Thickness of the three adrenal zones

\begin{tabular}{|c|c|c|c|c|c|c|}
\hline & \multicolumn{2}{|c|}{ ZG } & \multicolumn{2}{|c|}{ ZF } & \multicolumn{2}{|c|}{ ZR } \\
\hline & Mean \pm SD & $\mathbf{P}$ & Mean \pm SD & $\mathbf{P}$ & Mean \pm SD & $\mathbf{P}$ \\
\hline Group I-a & $51.7 \pm 6$ & \multirow{2}{*}{$0.02^{*}$} & $173.0 \pm 6.1$ & \multirow{2}{*}{$0.02^{*}$} & $115.0 \pm 5.0$ & \multirow{2}{*}{$0.003^{*}$} \\
\hline Group I-b & $41.3 \pm 5.5$ & & $190.7 \pm 5.5$ & & $127.3 \pm 5.1$ & \\
\hline Group II-a & $61.1 \pm 9.4$ & \multirow{2}{*}{0.074} & $167.3 \pm 6.4$ & \multirow{2}{*}{0.130} & $83 \pm 4.3$ & \multirow{2}{*}{0.069} \\
\hline Group II-b & $57.1 \pm 9.7$ & & $170.7 \pm 4.2$ & & $85.2 \pm 3.4$ & \\
\hline
\end{tabular}

${ }^{*}$ Significant $(\mathrm{p}<0.05)$; SD — standard deviation; ZG — zona glomerulosa; ZF — zona fasciculata; ZR — zona reticularis

engorgement of the central adrenal veins. The morphological changes under stress conditions adrenals developed against a background of haemodynamic changes that involved all layers of the adrenal cortex where the sinusoids and capillaries were dilated and plethoric [18].

Immunoreactivity to Ki67 was detected only in the stressed groups (groups I-b, II-b) as the cellular turnover in the adrenal cortex occurs only to accommodate the physiological needs or in response to experimental manipulations [23]. The pattern of distribution of immunoreactivity varied; in the adult stressed group, the reactivity was located in the subcapsular region, while in the senile stressed group it was confined to the junctional zone between ZG and ZF. The stem/progenitor cells exist in the adrenal capsule, subjacent cortex, and juxtamedullary region. These cells proliferate, differentiate, and repopulate in the cortex centripetally or centrifugally [23].

The ultrastructural remodelling of the adrenals of the stressed groups was mainly manifested as changes in the constituents involved in steroid hormone biosynthesis, namely, the lipid droplets, mitochondria, and smooth endoplasmic reticulum. The lipid droplets were seen packed together. These packed lipid droplets represent packets of steroid hormones awaiting release by exocytosis [8]. Other changes correlate with adrenal stimulation was the increasing number and size of mitochondria with more infolding of their inner membranes and their transformation to a more lamellar membrane pattern.

Another ultrastructural feature encountered in the senile stressed group was the degenerative changes of mitochondrial in the form of cristolysis, disruption of mitochondrial envelopes with the presence of intra-mitochondrial electron-dense inclusions [17]. The number of secondary lysosomes increased in this group which indicated autophagocytosis in the adrenocortical cells [19].
The senile stressed rats (group II-b) showed evident nuclear changes. The ZG cells exhibited nuclei with pyknosis, highly deformed irregular outlines and dense clumps of clogged chromatin. The ZF cells also showed highly indented bilobed nuclei with the undulation of the nuclear envelopes, electron-lucent spots and focal areas of chromatin destruction. These features of nuclear degeneration could reflect the superimposed effect of stress when coupled with ageing. The senile rats which were already exposed to the deleterious effect of ageing, showed marked deterioration upon stress exposure. Age prompted such effects through the affection of the hypothalamic-adrenocorticotropic-adrenal axis which may be confounded by multiple factors, including stress [26].

\section{CONCLUSIONS}

In conclusion, histological alterations occurred in the adrenal cortex in response to stress, especially when coupled with the advance of age. This was accompanied by proliferative changes.

\section{REFERENCES}

1. Bali A, Jaggi AS. Preclinical experimental stress studies: protocols, assessment and comparison. Eur J Pharmacol. 2015; 746: 282-292.

2. Balog M, Miljanović M, Blažetić S, et al. Sex-specific chronic stress response at the level of adrenal gland modified sexual hormone and leptin receptors. Croat Med J. 2015; 56(2): 104-113.

3. Becker KL. Principles and practice of endocrinology and metabolism. 3rd edn. Philadelphia: Lippincott Williams \& Wilkins. 2001

4. Brunk UT, Terman A. Lipofuscin-mechanisms of age-related accumulation and influence on cell function. Free Radic Biol Med. 2002; 33(5): 611-619.

5. Chang SP, Mullins JJ, Morley SD, et al. Transition from organogenesis to stem cell maintenance in the mouse adrenal cortex. Organogenesis. 2011; 7(4): 267-280.

6. da Silva Bi, Lopes-Costa PV, dos Santos AR, et al. Evaluation of Ki-67 antigen expression in the zona reticularis of the adrenal cortex of female rats in persistent estrus. Hum Reprod. 2009; 24(3): 705-709. 
7. Foilb AR, Lui P, Romeo RD. The transformation of hormonal stress responses throughout puberty and adolescence. J Endocrinol. 2011; 210(3): 391-398.

8. Gannouni N, Mhamdi A, El May M, et al. Morphological changes of adrenal gland and heart tissue after varying duration of noise exposure in adult rat. Noise Health. 2014; 16(73): 416-421.

9. Greaves P. Histopathology of preclinical toxicity studies: interpretation and relevance in drug safety evaluation. 4th edn. Amsterdam: Elsevier AP. 2012.

10. Hayashi $T$, Ikematsu $K$, Abe $Y$, et al. Temporal changes of the adrenal endocrine system in a restraint stressed mouse and possibility of postmortem indicators of prolonged psychological stress. Leg Med (Tokyo). 2014; 16(4): 193-196.

11. Hedrich HJ, Bullock GR. The laboratory mouse. Amsterdam; Oxford: Elsevier Academic Press. 2004.

12. Hornsby PJ. Aging of the human adrenal cortex. Sci Aging Knowledge Environ. 2004; 2004(35): re6, doi: 10.1126/ sageke.2004.35.re6, indexed in Pubmed: 15342924.

13. Hornsby PJ. Adrenarche: a cell biological perspective. J Endocrinol. 2012; 214(2): 113-119, doi: 10.1530/JOE12-0022, indexed in Pubmed: 22573830.

14. Janjua MZ, Khan MY. Age related changes in the rat adrenal cortex. J Pak Med Assoc. 1992; 42(4): 89-94.

15. Kim JS, Kubota H, Nam SY, et al. Expression of cytokines and proteases in mast cells in the lesion of subcapsular cell hyperplasia in mouse adrenal glands. Toxicol Pathol. 2000; 28(2): 297-303.

16. Koko V, Djordjeviae J, Cvijiae G, et al. Effect of acute heat stress on rat adrenal glands: a morphological and stereological study. J Exp Biol. 2004; 207(Pt 24): 4225-4230.

17. Koldysheva EV, Lushnikova EL. Ultrastructural reorganization of rat adrenal cortex after whole body hyperthermia. Bull Exp Biol Med. 2008; 145(5): 650-655.

18. Koldysheva EV, Lushnikova EL, Nepomnyashchikh LM. Morphogenesis of adaptation and compensatory reactions in mouse adrenals during restitution after thermal exposure. Bull Exp Biol Med. 2005; 140(4): 464-467.

19. Koldysheva EV, Lushnikova E, Proskuriakova IS. [Ultrastructural reorganization of the adrenal cortex during hypoxia and its correction by nerobolil in rats]. Biull Eksp Biol Med. 1999; 127(5): 588-592.

20. Krachulec J, Vetter M, Schrade A, et al. GATA4 is a critical regulator of gonadectomy-induced adrenocortical tumorigenesis in mice. Endocrinology. 2012; 153(6): 2599-2611.

21. Laast VA, Larsen $T$, Allison $N$, et al. Distinguishing cystic degeneration from other aging lesions in the adrenal cortex of Sprague-Dawley rats. Toxicol Pathol. 2014; 42(5): 823-9.

22. McEwen BS. Physiology and neurobiology of stress and adaptation: central role of the brain. Physiol Rev. 2007; 87(3): 873-904, doi:10.1152/physrev.00041.2006, indexed in Pubmed: 17615391.

23. Pihlajoki M, Dörner J, Cochran RS, et al. Adrenocortical zonation, renewal, and remodeling. Front Endocrinol (Lausanne). 2015; 6: 27, doi:10.3389/fendo.2015.00027, indexed in Pubmed: 25798129.

24. Pérez M, Terreros G, Dagnino-Subiabre A. Long-term $\omega$-3 fatty acid supplementation induces anti-stress effects and improves learning in rats. Behav Brain Funct. 2013; 9: 25.

25. Sahota PS. Toxicologic pathology: nonclinical safety assessment. Boca Raton CRC Press. 2013.

26. Veldhuis JD, Sharma A, Roelfsema F. Age-dependent and gender-dependent regulation of hypothalamic-adrenocorticotropic-adrenal axis. Endocrinol Metab Clin North Am. 2013; 42(2): 201-225.

27. Wass JA, Stewart PM. xford textbook of endocrinology and diabetes. 2nd edn. Oxford: Oxford University Press. 2011.

28. Yates R, Katugampola H, Cavlan D, et al. Adrenocortical development, maintenance, and disease. Curr Top Dev Biol. 2013; 106: 239-312, doi:10.1016/B978-0-12-416021-7. 00007-9, indexed in Pubmed: 24290352. 